\title{
Viscoelastic Modeling of Human Nasal Tissues with a Mobile Measurement Device
}

\author{
O. Işıtman ${ }^{1}$, O. Ayit ${ }^{1}$, E. Vardarlı ${ }^{1}$, Ş. Hanalioğlu ${ }^{2}$, İ. Işıkay ${ }^{2}$, M. Berker ${ }^{2}$, M. İ. C \\ Dede $^{1}$
}

\begin{abstract}
Modeling the dynamic of tool-tissue interaction for the robotic minimally invasive surgeries is one of the main issues for designing appropriate robot controllers. A mobile measurement device is produced in order to model some nasal tissues of a human. This mobile device is a hand-held one which measures the applied moments and relative angular displacements about a fixed pivot point. The ex-vivo measurements are realized by surgeons on a relatively fresh human cadaver head. The tip of the nose and the nasal concha are the two tissues that are investigated. In this study, five different viscoelastic models are considered; Elastic, KelvinVoight, Kelvin-Boltzmann, Maxwell and Hunt-Crossley. The results are evaluated and cross-validated on each data set. Hunt-Crossley and Kelvin-Boltzmann models provided the minimum root-mean-square (RMS) error among the other models.
\end{abstract}

Key words: Soft tissue modeling, Ex-vivo tests, Nasal tissue, Elastic model, KelvinVoight model, Kelvin-Boltzmann model, Maxwell model, Hunt-Crossley model

\section{Introduction}

In the past years, many open surgery procedures have been replaced by minimally invasive surgeries (MIS) and in the past three decades, robots started to be included in MIS. Introduction of robotics in MIS offered various benefits such as increased dexterity, increased manipulation capability and repeatability. However, one critical consideration in such procedures is the safety.

To avoid excessive contact force to the environment, the robotic surgical instrument must behave compliantly.A pure position control is not sufficient to solve this problem alone, hence, control of the dynamic interaction is also required [4]. An accurate model of the surgical environment, which happens to a soft tissue most of the case, can result in more precise and reliable controller design. Previously, a force control method was proposed based on pure elastic interaction in [12]. However, this assumption is not realistic for the biological tissues [3].

\footnotetext{
${ }^{1}$ Izmir Institute of Technology, Izmir, Turkey,

e-mail: \{ogulcanisitman, orhanayit, erenvardarli, candede\}@iyte.edu.tr

${ }^{2}$ Hacettepe University, Ankara, Turkey

e-mail: \{ilkayisikay, sahinhanalioglu\}@gmail.com

e-mail: mberker@hacettepe.edu.tr
} 
In order to define the tool-tissue interaction relatively more accurately, researchers have proposed several soft tissue models. These approaches can be classified as linear elasticity or nonlinear (hyperelastic) elasticity based finite element methods (FEM), viscoelastic models, fractional models, and high order models [7]. These models can also be classified by their computational time and deformation accuracy [9]. In the case of the diagnostic and scientific analysis of the tissue, more precise FEM's are suitable in spite of their longer computational time. Due to real-time requirement, analytical models are commonly used in the robotic applications. A conventional way to represent the tissue dynamics is using combinations of ideal damper and spring components. These models are comprehensively studied and compared in literature and schematic representation of some of these models are given in Fig. 1 along with their governing equations $[10,7,6]$. In the equations $F(t)$ and $x(t)$ represent the applied force and the deformation of tissue, respectively. $\beta=\frac{k_{1} k_{2}}{k_{1}+k_{2}}, \alpha=\frac{b k_{2}}{k_{1}+k_{2}}$ and $\delta=\frac{b}{k_{1}+k_{2}}$ are the linear combination of spring and damper parameters in the Kelvin-Boltzmann model.
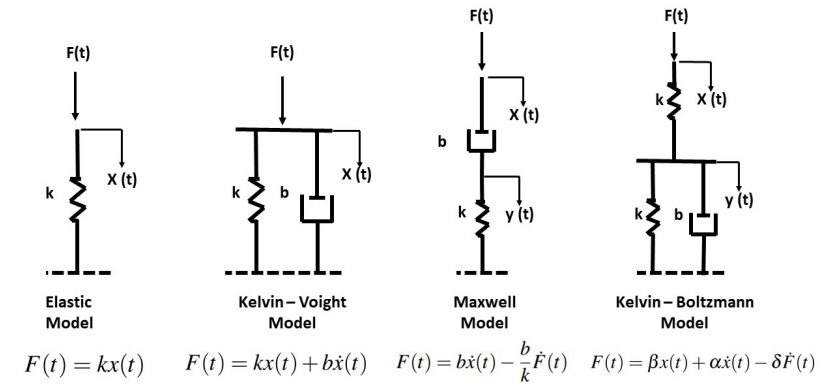

Fig. 1 Commonly used analytical models

The simplest model is a purely elastic model with single ideal spring model. The Kelvin-Voight model is consist of a parallel connection of an ideal damper and an ideal spring. On the other hand, the Maxwell model is composed of a serial connection of an ideal spring and an ideal damper components. Serial combination of elastic and the Kelvin-Voight models describes the Kelvin-Boltzmann model. These models differ from each other by their ability to mimic tissue behaviors such as creep and relaxation. These are the two major phenomena of the biological tissues [3]. While Maxwell model provides acceptable results for relaxation, Kelvin-Voight provides a good first order approximation of creep. On the other hand, Kelvin-Boltzmann model provides a good approximation for both creep and stress relaxation[7].

In [5], Hunt and Crossley presented that linear elastic models are not consistent with physics of contact and they proposed a non-linear model which is represented mathematically in eqn. 1. 


$$
F(t)=k x^{n}(t)+\lambda x^{n}(t) \dot{x}(t)
$$

In this equation, $n$ is positive scalar it ranges from 1.1 to 1.3 for a soft tissue. $[2,10,9]$. Several studies have experimentally showed that Kelvin-Boltzmann and Hunt-Crossley models provide relatively more realistic results with the lowest errors with respect to the aforementioned viscoelastic models [9].

In order to identify soft tissue model parameters, several types of experiments are performed in the literature. In most of these studies, a robotic system is used by driving an end-effector in a controlled manner into the in-vitro specimen [8].

The work presented in this paper is conducted to model the nasal tissues to be used in the design of an endoscopic pituitary tumor robotic surgery system. The conceptual design is presented in Fig. 2. In this robotic system, the robot is used to manipulate the endoscope inside the surgical area by rotating about a remote center of motion, which is usually called the pivot. Therefore, the endoscope handled by the robot is expected to interact with the nose tip and the nasal concha by making rotational motion.

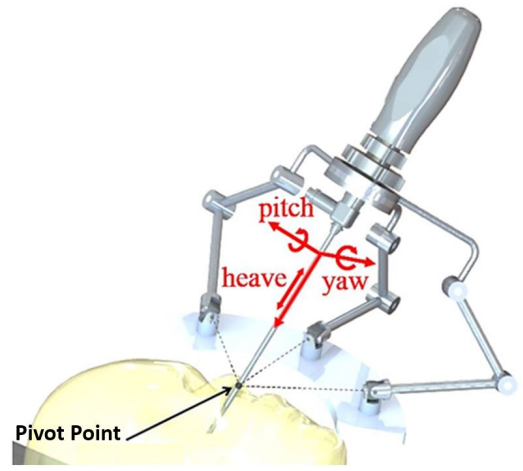

Fig. 2 Conceptual design of the robotic pituitary gland surgery system

Since preparing in-vitro specimen for this specific surgery area is not straightforward, we designed a new mobile hand-held measurement device which gives opportunity to acquire the necessary data in an ex-vivo set-up without the need of a robotic interface. However, the surgeon's hand motion cannot imitate a pure step input. For this reason, the response of soft tissue under compression is considered by moments applied on it for the evaluation instead of evaluating interaction models by their stress relaxation and creep behavior $[11,1]$. The soft tissue modeling is realized by using moment and angular displacement measurements due to the characteristic of the surgery which is another novelty of the study. Measurements are acquired from a relatively fresh human cadaver head in ex-vivo conditions.

In the next section, the proposed experimental set-up and test procedures are explained. In section 3, obtained results are presented and we discuss the results in the final section. 


\section{Methodology}

In order to acquire realistic data from the nasal tissue, a new mobile device is designed. An ATI MINI45 (ATI Industrial Automation) force/torque (F/T) sensor is used for the force and torque measurements and BNO055 (BOSCH Sensortech) inertial measurement unit (IMU) sensor is used to obtain angular displacements. Data acquisition is realized via MATLAB/Simulink environment with $100 \mathrm{~Hz}$ sampling rate. The components of the experimental set-up is presented in Fig. 3 indicating the measurement axes for angular motion and moment measurements.

The moment measurements are highly sensitive to the orientation of the device due to the weight of the F/T sensor. In order to eliminate gravitational forces, gravity compensation algorithm is developed by acquiring the absolute orientation of the device from the IMU sensor. These measurements also depend on the inertial forces and angular acceleration. To obtain more precise results, in the measurement process, the surgeons are asked to rotate the device at a constant velocity.

In order to imitate the real surgical scenario, a KARL STORZ telescope (28731 BWA Hopkins) is used and 3D printed holder is designed to provide better ergonomics for surgeons.

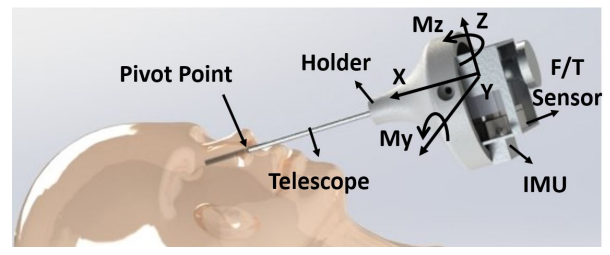

Fig. 3 CAD drawing of the proposed measurement device

Freshly frozen cadaver head is started to be thawed 48 hours beforehand to prepare for the experiment. Thin section tomography images of the cadaver head are taken with the Siemens Somamtom Perspective device during the thawing process. When this procedure is completed and the tissues became closer to the live tissue strength. The cadaver head is then fixed with a three-point head clamp (Mayfield, Integra Life Sciences Co., NJ) and the measurement protocols are applied.

Two test procedures are defined to measure applied moment and angular position data. In the first test, surgeons are asked to hold the device similar to the endoscope and perform the rotational movement around the predefined pivot point and compress the tip of the nose with a constant velocity. The tip of the nose is the actual pivot point in the endoscopic pituitary tumor surgery and the endoscope is expected to be in contact with this point during the operation. In the second test, surgeons are asked to rotate the device around the tip of the nose and compress the nasal concha without interacting with pivot point (no force applied to the nose tip).

Parameter estimation process is realized as follows: (1) Each interaction model is developed in Matlab Simulink by using angular position data and the resultant moment is compared with the measured moment data to obtain the error in the model. (2) The sum of the squares of error function is minimized by using fmincon 
function in Matlab. (3) The results are evaluated by using root-mean-square errors (RMSE) and using the average of the estimated parameters' cross-validation is realized for each measurement.

\section{Results}

The procedure that is explained in the previous section is realized by three surgeons that have carried out endoscopic pituitary tumor surgery. A total of nine experiments carried out in order to eliminate measurement error. Four of these experiments are performed to model the tip of the nose while the others were performed for the nasal concha model.

Table 1 Identified Parameters for the Tip of the Nose Models)

\begin{tabular}{|c|c|c|c|c|c|}
\hline & & \multicolumn{4}{|c|}{ Experiments } \\
\hline Models & & $\# 1$ & $\# 2$ & $\# 3$ & $\# 4$ \\
\hline \multirow{3}{*}{ Kelvin Boltzmann } & $k_{2}$ & 0,455 & 1,208 & 0,860 & 1,363 \\
& $k_{1}$ & 0,135 & 0,375 & 0,273 & 0,149 \\
& $b$ & 16.270 & 28,457 & 24,695 & 25,105 \\
\hline \multirow{2}{*}{ Kelvin Voight } & $k$ & 0,102 & 0,276 & 0,202 & 0,239 \\
& $b$ & 0,209 & 0,115 & 0,448 & 0,593 \\
\hline Elastic & $\mathrm{k}$ & 0,103 & 0,277 & 0,202 & 0,247 \\
\hline \multirow{2}{*}{ Maxwell } & $\mathrm{k}$ & 0,589 & 1,469 & 1,216 & 1,243 \\
& $\mathrm{~b}$ & 101,309 & 125,209 & 127,071 & 127,302 \\
\hline \multirow{2}{*}{ Hunt Crossley } & $\mathrm{k}$ & 0,127 & 0,358 & 0,308 & 0,327 \\
& $\lambda$ & 15,154 & 15,173 & 15,494 & 15,492 \\
\hline
\end{tabular}

The measured position/torque values in the experiments are used to calculate the parameters of soft tissue models. In each experiment, the maximum measured torque is approximately $0.03 \mathrm{Nm}$.

Table 1 shows the identified parameters of the nose tip models for each experiment where the unit of spring $\left(k, k_{1}, k_{2}\right)$ and damper $(b)$ parameters are $\mathrm{Nm} / \mathrm{rad}$ and $N m . s / r a d$, respectively. It is observed in Table 1 that the parameters are consistent with each other.

Table 2 RMSE between Measured and Calculated Moments for the Nose Tip Models

\begin{tabular}{|c|c|c|c|c|}
\hline \multicolumn{5}{|c|}{ RMSE (Nm) } \\
\hline & \multicolumn{4}{|c|}{ Experiments } \\
\hline Models & $\# 1$ & $\# 2$ & $\# 3$ & $\# 4$ \\
\hline Kelvin Boltzmann & $\mathbf{0 , 0 0 3 6}$ & 0,0056 & $\mathbf{0 , 0 0 5 8}$ & $\mathbf{0 , 0 0 5 1}$ \\
\hline Kelvin Voight & $\mathbf{0 , 0 0 3 6}$ & 0,0057 & 0,0059 & 0,0056 \\
\hline Elastic & $\mathbf{0 , 0 0 3 6}$ & 0,0056 & 0,0059 & 0,0058 \\
\hline Maxwell & 0,0043 & 0,0069 & 0,0067 & 0,0056 \\
\hline Hunt Crossley & 0,0046 & $\mathbf{0 , 0 0 5 3}$ & 0,0060 & 0,0074 \\
\hline
\end{tabular}

In addition, RMSE between measured torques and calculated ones are reported in Table 2 and the minimum RMSE value received for the model of each experiment 
is highlighted with bold fonts. It is observed that the Kelvin-Boltzmann model resulted in the minimal errors in experiments \#1,\#3, \#4 and Hunt-Crossley model has minimal error in experiment \#2. Also, Kelvin-Voight and Elastic models resulted in the same minimal error in experiment \#1.

Table 3 RMSE between Measured and Calculated Force with Average Parameters of the Nose Tip Models

\begin{tabular}{|c|c|c|c|c|c|c|}
\hline & & \multicolumn{5}{|c|}{ Experiments } \\
\hline & & \multicolumn{4}{|c|}{ RMSE (Nm) } \\
\hline Models & & Avg. Coeff. & $\# 1$ & $\# 2$ & $\# 3$ & $\# 4$ \\
\hline \multirow{2}{*}{ Kelvin Boltzmann } & $k_{2}$ & 0,972 & & & & \\
& $k_{1}$ & 0,233 & $\mathbf{0 , 0 2 1 6}$ & 0,0165 & $\mathbf{0 , 0 0 6 2}$ & 0,0111 \\
& $b$ & 23,631 & & & & \\
\hline \multirow{2}{*}{ Kelvin Voight } & $k$ & 0,205 & 0,0235 & 0,0156 & $\mathbf{0 , 0 0 6 2}$ & 0,0108 \\
& $b$ & 0,341 & & & & \\
\hline Elastic & $\mathrm{k}$ & 0,207 & 0,0237 & 0,0155 & $\mathbf{0 , 0 0 6 2}$ & 0,0114 \\
\hline \multirow{2}{*}{ Maxwell } & $\mathrm{k}$ & 1,129 & 0,0217 & 0,0135 & 0,0068 & 0,0099 \\
& $\mathrm{~b}$ & 120,223 & & & & \\
\hline \multirow{2}{*}{ Hunt Crossley } & $\mathrm{k}$ & 0,280 & 0,0264 & $\mathbf{0 , 0 1 3 2}$ & 0,0064 & $\mathbf{0 , 0 0 9 6}$ \\
& $\lambda$ & 15,328 & & & & \\
\hline
\end{tabular}

The average values of identified parameters of the models are calculated. The soft tissue models with these new parameters are evaluated for each experiment for cross-validation and the RMSE values are calculated as shown in Table 3. According to these results, the minimum RMSE value is obtained when Kelvin-Boltzmann, Kelvin Voight, and Elastic models are applied to experiment \#3. Also, the KevinBoltzmann has minimum RMSE value in experiment \#1. In experiment \#2 and \#4, Hunt-Crossley model has the best performance.

Table 4 Identified Parameters for the Nasal Concha Model

\begin{tabular}{|c|c|c|c|c|c|c|}
\hline & & \multicolumn{5}{|c|}{ Experiments } \\
\hline Models & & $\# 1$ & $\# 2$ & $\# 3$ & $\# 4$ & $\# 5$ \\
\hline \multirow{3}{*}{ Kelvin Boltzmann } & $k_{2}$ & 3,829 & 3,700 & 4,198 & 2,993 & 1,795 \\
& $k_{1}$ & 0,036 & 0,559 & 1,033 & 0,207 & 0,559 \\
& $b$ & 26,357 & 32,038 & 33,878 & 29,029 & 30,979 \\
\hline \multirow{2}{*}{ Kelvin Voight } & $k$ & 0,575 & 0,675 & 0,844 & 0,419 & 0,358 \\
& $b$ & 0,733 & 0,236 & 1,525 & 1,773 & 0,517 \\
\hline Elastic & $\mathrm{k}$ & 0,600 & 0,679 & 0,856 & 0,440 & 0,370 \\
\hline \multirow{2}{*}{ Maxwell } & $\mathrm{k}$ & 2,928 & 3,440 & 5,833 & 2,545 & 2,632 \\
& $\mathrm{~b}$ & 137,166 & 136,950 & 139,842 & 136,699 & 138,644 \\
\hline \multirow{2}{*}{ Hunt Crossley } & $\mathrm{k}$ & 1,000 & 1,070 & 1,586 & 0,824 & 0,787 \\
& $\lambda$ & 15,038 & 14,990 & 15,10 & 15,117 & 15,535 \\
\hline
\end{tabular}

Similar studies are carried out to model the nasal concha. Table 4 presents the identified parameters and it is observed that the parameters are consistent with each other. Also, RMSE values of each model are given in Table 5 and the minimum- 
error ones are highlighted by bold fonts. According to Table 5, Kelvin-Boltzmann has the best performance in experiment \#1, \#2, \#3 and \#4. In experiment \#5, the minimum error is obtained when Hunt-Crossley model is applied.

Table 5 RMSE between Measured and Calculated Force for the Nasal Concha Models

\begin{tabular}{|c|c|c|c|c|c|}
\hline \multicolumn{5}{|c|}{ RMSE (Nm) } \\
\hline & \multicolumn{5}{|c|}{ Experiments } \\
\hline Models & $\# 1$ & $\# 2$ & $\# 3$ & $\# 4$ & $\# 5$ \\
\hline Kelvin Boltzmann & $\mathbf{0 , 0 0 3 9}$ & $\mathbf{0 , 0 0 4 8}$ & $\mathbf{0 , 0 0 3 4}$ & $\mathbf{0 , 0 0 2 4}$ & 0,0031 \\
\hline Kelvin Voight & 0,0072 & 0,0068 & 0,0035 & 0,0033 & 0,0033 \\
\hline Elastic & 0,0075 & 0,0068 & 0,0035 & 0,0036 & 0,0030 \\
\hline Maxwell & 0,0068 & 0,0050 & 0,0050 & 0,0036 & 0,0059 \\
\hline Hunt Crossley & 0,0128 & 0,0111 & 0,0049 & 0,0050 & $\mathbf{0 , 0 0 2 9}$ \\
\hline
\end{tabular}

To obtain an approximate tissue model for each experiment, the averages of the models' parameters are calculated and these values are used for each experiment for cross-validation. The average parameters and the RMSE values for model validation are presented in Table 6 .

Table 6 RMSE between Measured and Obtained Force with Average Parameters of the Nasal Concha Models

\begin{tabular}{|c|c|c|c|c|c|c|c|}
\hline & & \multicolumn{6}{|c|}{ Experiments } \\
\hline & & & & & MSE $(\mathrm{Nr}$ & & \\
\hline Models & & Avg. Coeff. & $\# 1$ & $\# 2$ & \#3 & $\# 4$ & $\# 5$ \\
\hline Kelvin Boltzmann & $\begin{array}{l}k_{1} \\
b\end{array}$ & $\begin{array}{c}3,303 \\
0,479 \\
30,456\end{array}$ & 0,0157 & 0,0085 & 0,0128 & 0,0055 & 0,0080 \\
\hline Kelvin Voight & 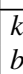 & $\begin{array}{l}0,574 \\
0,957\end{array}$ & 0,0198 & 0,0114 & 0,0106 & 0,0064 & 0,007 \\
\hline Elastic & $\mathrm{k}$ & 0,589 & 0,0206 & 0,0110 & 0,0104 & 0,0066 & 0,0068 \\
\hline Maxwell & $\mathrm{k}$ & $\begin{array}{c}3,476 \\
137,860\end{array}$ & 0,0334 & 0,0111 & 0,0107 & 0,0083 & 0,0098 \\
\hline Hunt Crossley & & $\begin{array}{c}1,054 \\
15,158\end{array}$ & 0,0237 & 0,0050 & 0,0117 & 0,0068 & 0,0048 \\
\hline
\end{tabular}

According to these values, Kevin-Boltzmann model has the best performance in experiment \#1 and \#4. In experiment \#2 and \#5, the minimum error is observed when Hunt-Crossley model is applied. In contrast to other experiments, the elastic model has the best performance in experiment \#3.

\section{Discussions and Conclusions}

In the endoscopic pituitary tumor surgery, the tip of nose and tissue of the nasal concha mostly interacts with the endoscope. Since the results of this modeling study will 
be used in the design of a remote center of motion kinematics based robotic surgery, this study investigated the behavior of tissues under rotational movement which is different from the most of the works in literature. We proposed a new mobile measurement device to acquire the torque/position data during the interactions with the focused soft tissues of a cadaver in ex-vivo tests. In the experiments, three surgeons are asked to apply torques to compress tissues with a constant angular velocity.

The soft tissues models for the nasal concha and the nose tip are obtained by suing the experimental data. The results meet the expectations since the other studies in the literature show that the Kelvin-Boltzmann and Hunt-Crossley models have better performance relative to the others.

As a result, this study provides quantitative knowledge about the expected torques during the operation and an accurate interaction model in order to design a complaint controller as a future work.

Acknowledgements This work is supported by The Scientific and Technological Research Council of Turkey via grant numbers 115E725 and 115E726.

\section{References}

1. BAO, Y., WU, D., YAN, Z., AND DU, Z. A new hybrid viscoelastic soft tissue model based on meshless method for haptic surgical simulation. The open biomedical engineering journal 7 (2013), 116.

2. Diolaiti, N., Melchiorri, C., and Stramigioli, S. Contact impedance estimation for robotic systems. IEEE Transactions on Robotics 21, 5 (2005), 925-935.

3. FUNG, Y., AND SKALAK, R. Biomechanics. mechanical properties of living tissues. Journal of Applied Mechanics 49 (1982), 464.

4. HogAn, N. Impedance control: An approach to manipulation. In American Control Conference, 1984 (1984), IEEE, pp. 304-313.

5. Hunt, K., And Crossley, F. Coefficient of restitution interpreted as damping in vibroimpact. Journal of applied mechanics 42, 2 (1975), 440-445.

6. LiU, C., Moreira, P., And Poignet, P. Viscoelastic model based force tracking control for robotic-assisted surgery. In Biomedical Robotics and Biomechatronics (BioRob), 2012 th IEEE RAS \& EMBS International Conference on (2012), IEEE, pp. 1199-1204.

7. Misra, S., Ramesh, K., AND OKamura, A. M. Modeling of tool-tissue interactions for computer-based surgical simulation: A literature review. Presence: Teleoperators and Virtual Environments 17, 5 (2008), 463-491.

8. Moreira, P., LiU, C., Zemiti, N., And Poignet, P. Soft tissue force control using active observers and viscoelastic interaction model. In Robotics and Automation (ICRA), 2012 IEEE International Conference on (2012), IEEE, pp. 4660-4666.

9. Moreira, P., Zemiti, N., LiU, C., And Poignet, P. Viscoelastic model based force control for soft tissue interaction and its application in physiological motion compensation. Computer methods and programs in biomedicine 116, 2 (2014), 52-67.

10. Pappalardo, A., Albakri, A., Liu, C., Bascetta, L., De Momi, E., And Poignet, P. Hunt-crossley model based force control for minimally invasive robotic surgery. Biomedical Signal Processing and Control 29 (2016), 31-43.

11. Takács, Á., Galambos, P., Pausits, P., Rudas, I. J., and Haidegger, T. Nonlinear soft tissue models and force control for medical cyber-physical systems. In Systems, Man, and Cybernetics (SMC), 2015 IEEE International Conference on (2015), IEEE, pp. 1520-1525.

12. Yuen, S. G., Perrin, D. P., Vasilyev, N. V., Pedro, J., and Howe, R. D. Force tracking with feed-forward motion estimation for beating heart surgery. IEEE Transactions on Robotics 26, 5 (2010), 888-896. 